\title{
CEA-MUC-1-TRICOM Vaccine CV301
}

National Cancer Institute

\section{Source}

National Cancer Institute. CEA-MUC-1-TRICOM Vaccine CV301. NCI Thesaurus. Code C131129.

A cancer prime/boost vaccine-based immunotherapeutic consisting of a prime, which is comprised of a proprietary version of the recombinant vaccinia viral vector, modified vaccinia Ankara-Bavarian Nordic (MVA-BN) and a recombinant fowlpox viral vector, used for the boosts, encoding both the two tumor-associated antigens (TAA),

carcinoembryonic antigen (CEA) and mucin-1 (MUC-1), and TRICOM, which is comprised of three immune-enhancing co-stimulatory molecules, B7-1, ICAM-1 and LFA-3, with potential immunostimulatory and antineoplastic activities. The administration of the vaccinia priming dose is followed by multiple boosting doses of the fowlpox vector. CEAMUC-1-TRICOM Vaccine CV301 may enhance presentation of CEA and MUC-1 to antigen-presenting cells (APCS) and may activate a cytotoxic T-lymphocyte (CTL) response against CEA- and MUC-1-expressing tumor cells. In addition, CV301 upregulates the expression of PD-L1 due to CT L-mediated tumor attack; additionally, when combined with a PD-1 immune checkpoint inhibitor, the antitumor effect may be increased. CEA and MUC-1 are overexpressed in certain cancers. 